\title{
An improved control-variate scheme for particle-in-cell simulations with collisions
}

\author{
R. Kleiber \\ Max-Planck-Institut für Plasmaphysik, EURATOM Association \\ 17491 Greifswald, Germany \\ R. Hatzky \\ Max-Planck-Institut für Plasmaphysik, EURATOM Association \\ 85748 Garching, Germany \\ A. Könies \\ Max-Planck-Institut für Plasmaphysik, EURATOM Association \\ 17491 Greifswald, Germany \\ K. Kauffmann \\ Max-Planck-Institut für Plasmaphysik, EURATOM Association \\ 17491 Greifswald, Germany \\ P. Helander \\ Max-Planck-Institut für Plasmaphysik, EURATOM Association \\ 17491 Greifswald, Germany
}

\begin{abstract}
Particle-in-cell methods combined with a $\delta f$ approach constitute an established and powerful method for simulating collisionless kinetic equations in e.g. plasma physics. Including collisions in such simulations requires a modified approach leading to a two-weight scheme, which has the drawback of giving a statistical error that increases with time. As in the collisionless case, this scheme can be interpreted as an application of an ordinary control variate. Using an enhanced control variate approach, an improved scheme is constructed. This approach has been applied to a model problem with the result of a much better behaviour of the error, which, instead of growing indefinitely, becomes bounded by the error of a full- $f$ scheme. In addition, the application of the enhanced control variate is illustrated for a collisionless simulation of ITG turbulence. Here it can be used both as a diagnostic tool and as a means to eliminate the spurious violation of particle number conservation inherent to $\delta f$ simulations due to statistical noise.
\end{abstract}

Keywords: particle-in-cell, Monte Carlo simulations, collisions 


\section{Introduction}

Particle-in-cell (PIC) methods [1, 2] are well established for solving kinetic equations in complicated geometries. The gyrokinetic equation [3] is a firstprinciples equation encompassing much of the physics which is necessary to describe the confinement of hot magnetised plasmas in the core region of fusion devices where, in a first approximation, the plasma can be regarded as collisionless. When modelling such a plasma, it is highly important to simulate ion-temperature-gradient driven modes since they can evolve into saturated turbulence leading to anomalous transport. Numerically, two methods are used for such simulations: The continuum approach, where the gyrokinetic equation is solved as a partial differential equation, and the particle-in-cell approach. Due to their flexibility and because they are relatively easy to parallelise, PIC methods have developed into a powerful means of simulating fusion plasmas. Nevertheless, their inherent drawback is the noise due to the particle discretisation which decreases with particle number $N$ only as $O(1 / \sqrt{N})$.

In fusion plasmas, the equilibrium state is usually assumed to be a Maxwellian velocity distribution with fluctuations driven by the presence of gradients in density and temperature. For a broad range of problems, the relative size of such perturbations can be about $O\left(10^{-2}\right)$ (or less). So, the time evolution of the system usually leads to new states that are relatively nearby to the initial state. Consequently, a very high number of markers would be necessary in order to make the particle noise smaller than the magnitude of the perturbation which needs to be resolved. Especially the linear phase of an instability, where the relative perturbation is even smaller by many orders of magnitude, would be practically inaccessible. Therefore, using a direct (full- $f$ ) approach would result in wasting most of the marker resolution to represent the Maxwellian and the perturbation would be endangered of being drowned by the noise. Having low noise becomes even more necessary since normally some moments of the distribution function have a back reaction on the evolution of the system.

These problems lead to the introduction of the so-called $\delta f$ approach [4] where the markers are used to simulate only the deviation from the initial state. This method has, since its conception, become the main method for gyrokinetic PIC simulations. Originally, it was introduced as a splitting of the distribution function into the sum of a time-independent part (assumed to be Maxwellian) and a time-dependent part being simulated. As a consequence, the markers have to carry a weight evolving in time according to a differential equation. Later, Aydemir [5] pointed out that in a Monte Carlo framework the $\delta f$ scheme is equivalent to a control variate scheme (solving the differential equation for the weight then becomes superfluous [6]). Using a control variate not only made the method more transparent, but also showed how it can be extended. This lead to the development of an adjustable control variate for electromagnetic simulations [7].

Even in the core region of a plasma, collisions can have an influence, e.g. neoclassical transport is purely collisional and trapped electron modes can be stabilised by collisions. Thus, it is necessary to extend the $\delta f$ method to include collisional 
effects. While for a full- $f$ approach it is relatively easy to find a simulation scheme, it is more difficult to find and justify a scheme in the $\delta f$ framework. A formal way of deriving such a scheme by working in an extended phase space was introduced in [8]. Unfortunately, this scheme was unpractical, and only after it had been extended to a two-weight scheme in $[9,10]$ a more practicable scheme resulted. Nevertheless, a major drawback of the latter scheme is that the statistical error increases with time (so-called weight-spreading) and computational expensive procedures are necessary to avoid this phenomenon [9].

Our paper is organised as follows: First, we state the problem and the relevant equations in a general way and give a transparent derivation of the usual two-weight scheme. Using a simple but useful example, the numerical behaviour of the scheme is demonstrated. We then show that, in an important special case, the scheme can be regarded as an ordinary control variate scheme. We then introduce an enhanced control variate and use it to construct a modified collisional scheme which shows a much better behaviour of the statistical error. The enhanced control variate approach is further illustrated by applying it to a collisionless simulation of turbulence in order to avoid the violation of particle number conservation.

\section{Equations}

In the following, we endeavour to use a particle-in-cell method to solve a kinetic equation of the type

$$
\frac{\mathrm{d} f}{\mathrm{~d} t}=\frac{\partial f}{\partial t}+\dot{z}^{i} \cdot \frac{\partial f}{\partial z^{i}}=C(f, f)+\mathcal{S},
$$

where the total time derivative is denoted by a dot, $f(\mathbf{z})$ is a distribution function in a six-dimensional phase space, $C(f, f)$ a collision operator and $\mathcal{S}$ a source term. For simplicity, the vector $\mathbf{z}=(\mathbf{R}, \mathbf{v})$ has been introduced which contains the position $\mathbf{R}$ and velocity $\mathbf{v}$ coordinates. The equations of motion are given by

$$
\dot{\mathbf{R}}=\mathbf{v}_{0}+\mathbf{v}_{1}, \quad \dot{\mathbf{v}}=\mathbf{a}_{0}+\mathbf{a}_{1}
$$

where $\mathbf{v}_{0}, \mathbf{a}_{0}$ are the unperturbed quantities which only depend on the timeindependent background and $\mathbf{v}_{1}, \mathbf{a}_{1}$ are the perturbed quantities which depend on, e.g. the electrostatic potential $\Phi$. It is further assumed that the flow given by Eq. (2) is incompressible: $\frac{1}{\mathcal{J}} \frac{\partial}{\partial z^{i}}\left(\dot{z}^{i} \mathcal{J}\right)=0$ with $\mathcal{J}$ being the Jacobian. The electrostatic potential is obtained from a field equation of the type $L \Phi=n$ where $L$ is an elliptic operator with appropriate boundary conditions and the density at position $\mathbf{x}$ is given as a moment of the distribution function

$$
n(\mathbf{x}, t)=\int f(\mathbf{z}, t) K(\mathbf{x}, \mathbf{z}) \mathrm{d} \mathbf{z}
$$

with a given kernel $K$. For example, the gyrokinetic equation [3] belongs to this type of equations and the kernel represents a gyro-averaging operation. For 
this case, the equations of motion follow from a Hamiltonian principle and thus inherently lead to an incompressible flow.

Using the incompressibility of the flow one arrives at the conservative form of Eq. (1)

$$
\frac{\partial f}{\partial t}+\frac{1}{\mathcal{J}} \frac{\partial}{\partial z^{i}}\left(\dot{z}^{i} \mathcal{J} f\right)=C(f, f)+\mathcal{S}
$$

The collision operator is assumed to be given by the familiar expression (see e.g. [11])

$$
C\left(f_{1}, f_{2}\right)=\frac{1}{\mathcal{J}} \frac{\partial}{\partial v^{i}}\left(-S^{i}\left(\mathbf{v}, f_{2}\right) \mathcal{J} f_{1}(\mathbf{v})+\frac{\partial}{\partial v^{j}}\left[D^{i j}\left(\mathbf{v}, f_{2}\right) \mathcal{J} f_{1}(\mathbf{v})\right]\right),
$$

where the drag vector $\mathbf{S}$ and the diffusion tensor $\mathbf{D}$ for the case of Coulomb collisions involve the Rosenbluth potentials [12]. The dependence of $\mathbf{S}$ and $\mathbf{D}$ on the distribution function makes the collision operator nonlinear, but for simplicity it is usually linearised.

As a remark, we note that in the gyrokinetic equation, due to the averaging over the fast gyration of the particle, the collision operator contains, in principle, diffusion both in velocity and position space [13] but is often simplified to Eq. (5) by keeping only the velocity part.

\section{Collisionless $\delta f$ method}

One method to solve Eqs. (1-3) for the collisionless case, $C=0$, is to use a $\delta f$ PIC approach [4]. For this approach, one introduces $N$ marker particles, distributed according to a marker distribution function $g(\mathbf{z}, t)$ which move along trajectories given by Eq. (2). A central point in PIC simulations is the calculation of moments of the distribution function (e.g. in order to calculate the density [Eq. (3)]), which are formulated as expectation values. Using the marker particles, a Monte Carlo estimator then approximates the expectation values:

$$
\int A(\mathbf{z}) f(\mathbf{z}) \mathrm{d} \mathbf{z} \approx \frac{1}{N} \sum_{p=1}^{N} w_{p} A\left(\mathbf{z}_{p}\right),
$$

where the weight $w_{p}=f\left(\mathbf{z}_{p}\right) / g\left(\mathbf{z}_{p}\right)$ has been introduced, $\mathbf{z}_{p}$ denotes the position of each marker $p$ in phase space and $A$ is an arbitrary function. The error of this estimate is $\mathcal{O}\left(\frac{1}{\sqrt{N}}\right)$ and should be as low as possible.

The distribution function is now written as $f(\mathbf{z}, t)=f_{0}(\mathbf{z})+\delta f(\mathbf{z}, t)$ with $f_{0}$ being a time-independent function and $\delta f$ a time-dependent perturbation. Substituting this into Eq. (1) (with $C=0, \mathcal{S}=0$ ) one obtains an evolution equation for $\delta f$

$$
\frac{\mathrm{d} \delta f}{\mathrm{~d} t}=\dot{z}^{i} \cdot \frac{\partial f_{0}}{\partial z^{i}} .
$$


Using the theorem of characteristics and the fact that $\frac{\mathrm{d} g}{\mathrm{~d} t}=0$ this gives an evolution equation for the weight of each marker [14]:

$$
\frac{\mathrm{d} w_{p}}{\mathrm{~d} t}=\frac{1}{g_{p}} \dot{z}_{p}^{i} \cdot \frac{\partial f_{0}}{\partial z_{p}^{i}} .
$$

The density perturbation follows from Eq. (3) as:

$$
\delta n=n-\int f_{0} K \mathrm{~d} \mathbf{z}=\int \delta f K \mathrm{~d} \mathbf{z} .
$$

Here, the integral over $f_{0}$ is known analytically while the integral over $\delta f$ is evaluated using the Monte Carlo estimator given by Eq. (6).

As Aydemir [5] showed, this procedure can be interpreted in the framework of a Monte Carlo approach by noting that it is equivalent to the introduction of $f_{0}$ as a control variate $(\mathrm{CV})$ in Eq. (3)

$$
n=\int\left(f-f_{0}\right) K \mathrm{~d} \mathbf{z}+\int f_{0} K \mathrm{~d} \mathbf{z}
$$

In the collisionless case (and with $\mathcal{S}=0) f$ and $g$ are conserved along the trajectories $\left(\frac{\mathrm{d} f}{\mathrm{~d} t}=0, \frac{\mathrm{d} g}{\mathrm{~d} t}=0\right)$ and thus, the weight can directly be obtained as

$$
w_{p}=c_{p}\left(t_{0}\right)-\frac{f_{0}\left(\mathbf{z}_{p}(t)\right)}{g\left(\mathbf{z}_{p}\left(t_{0}\right)\right)}, \quad c_{p}\left(t_{0}\right)=\frac{f\left(\mathbf{z}_{p}\left(t_{0}\right)\right)}{g\left(\mathbf{z}_{p}\left(t_{0}\right)\right)}
$$

(where $c_{p}\left(t_{0}\right)$ is a constant determined by the initial conditions at $t_{0}$ ) without the need of integrating Eq. (8) [6].

So, in a $\delta f$ simulation, each marker carries a weight $w_{p}$, which is a measure of its contribution to the distribution function $f$, while the markers are distributed according to a marker distribution function $g$ (that can be chosen independently from $f[15]$ ). It is only necessary to resolve the deviation of $f$ from $f_{0}$ by marker particles. This decreases the noise by several orders of magnitude, if $\delta f \ll f_{0}$ (as it is usually the case for microinstabilities in plasmas).

If collisions are included, $f$ and $g$ are no longer constants of the motion and the above procedure cannot be used. Thus, one needs a modified method that still separates the large time-independent part $f_{0}$ from $f$. Such a method was first introduced in [8] and later extended by introducing a second weight $[9,10]$. Using these ideas, we derive the collisional two-weight scheme in a general setting.

\section{Derivation of the collisional scheme}

As the basis of the derivation, we use the following theorem (see e.g. [16, 17]): The solution of the $n$-dimensional Kolmogorov forward (or Fokker-Planck) equation

$$
\frac{\partial}{\partial t} f\left(x^{1} \ldots x^{n}, t\right)=-\frac{1}{\mathcal{J}} \frac{\partial}{\partial x^{i}}\left[\mu^{i} \mathcal{J} f\right]+\frac{1}{2 \mathcal{J}} \frac{\partial^{2}}{\partial x^{i} \partial x^{j}}\left[D^{i}{ }_{k} D_{l}^{j} \delta^{k l} \mathcal{J} f\right]
$$


is equivalent to the probability distribution function for the solutions of the (Itoh) stochastic differential equations

$$
\mathrm{d} x^{i}=\mu^{i} \mathrm{~d} t+D_{j}^{i} \mathrm{~d} W^{j} .
$$

Here, $\mu_{i}, D^{i}{ }_{j}, W^{j}$ are the drift coefficients, the diffusion matrix and an $n$-dimensional Wiener process, respectively.

Writing, as before, $f$ in Eq. (1) as $f=f_{0}+\delta f$ and linearising the collision operator by neglecting $C(\delta f, \delta f)$, one obtains

$$
\mathcal{P} \delta f=C\left(f_{0}, f_{0}\right)+C\left(f_{0}, \delta f\right)+\mathcal{S}-\mathcal{S}_{1},
$$

where $\mathcal{P} \delta f \stackrel{\text { def }}{=} \frac{\partial}{\partial t} \delta f+\frac{1}{\mathcal{J}} \frac{\partial}{\partial z^{i}}\left(\dot{z}^{i} \mathcal{J} \delta f\right)-C\left(\delta f, f_{0}\right)$ and $\mathcal{S}_{1}=\dot{z}^{i} \frac{\partial f_{0}}{\partial z^{i}}$ have been introduced. This is to be solved by using a Monte Carlo approach. Note that the operator $C\left(f_{0}, \delta f\right)$ is a very complicated one, since the unknown $\delta f$ appears in the Rosenbluth potentials leading to an integro-differential equation. This operator ensures momentum and energy conservation and can be important in some applications, but it is then usually replaced by a simplified model $[13,18]$. Using Eq. (5), the left-hand side of Eq. (14) can be seen to have the form of Eq. (12). Nevertheless, the basic theorem [Eqs. $(12,13)]$ cannot be used because the right-hand side is not zero. To circumvent this problem one introduces an extended phase space $\left(\mathbf{R}, \mathbf{v}, w_{1}, w_{2}\right)$ where $w_{1}, w_{2}$ are two additional dimensions (here we follow Ref. [9]) being interpreted as weights. In this extended phase space, the following evolution equation for an (extended) distribution function $F\left(\mathbf{R}, \mathbf{v}, w_{1}, w_{2}\right)$ is postulated

$$
\hat{\mathcal{P}} F+\frac{1}{\hat{\mathcal{J}}} \frac{\partial}{\partial w_{1}}\left(A_{1} \hat{\mathcal{J}} F\right)+\frac{1}{\hat{\mathcal{J}}} \frac{\partial}{\partial w_{2}}\left(A_{2} \hat{\mathcal{J}} F\right)=0 .
$$

$\hat{\mathcal{J}}$ denotes the Jacobian in the extended phase space, $\hat{\mathcal{P}}$ is the same as $\mathcal{P}$ but with $\mathcal{J}$ replaced by $\hat{\mathcal{J}}$ and $A_{1}, A_{2}$ are free quantities which are specified later. Identifying $x^{1} \ldots x^{8}$ with $\mathbf{R}, \mathbf{v}, w_{1}, w_{2}$ and, following from this, making obvious choices for $\mu_{i}$ and $D^{i}{ }_{j}$, Eq. (15) can be written in the form of Eq. (12) and thus allows an application of the basic theorem. The set of stochastic differential equations for each marker $p$ is then

$$
\begin{aligned}
\dot{\mathbf{R}}_{p} & =\mathbf{v}_{0}+\mathbf{v}_{1} \\
\dot{\mathbf{v}}_{p} & =\mathbf{a}_{0}+\mathbf{a}_{1}+\mathbf{S}(\mathbf{v})+\mathbf{D}(\mathbf{v}) \cdot \frac{\mathrm{d} \mathbf{W}}{\mathrm{d} t} \\
\dot{w}_{1, p} & =A_{1}, \dot{w}_{2, p}=A_{2} .
\end{aligned}
$$

Furthermore, the following moments of $F$ are defined:

$$
\begin{aligned}
g(\mathbf{R}, \mathbf{v}) & \stackrel{\text { def }}{=} \frac{1}{\mathcal{J}} \int F \hat{\mathcal{J}} \mathrm{d} w_{1} \mathrm{~d} w_{2} \\
u(\mathbf{R}, \mathbf{v}) & \stackrel{\text { def }}{=} \frac{1}{\mathcal{J}} \int w_{1} F \hat{\mathcal{J}} \mathrm{d} w_{1} \mathrm{~d} w_{2} \\
h(\mathbf{R}, \mathbf{v}) & \stackrel{\text { def }}{=} \frac{1}{\mathcal{J}} \int w_{2} F \hat{\mathcal{J}} \mathrm{d} w_{1} \mathrm{~d} w_{2} .
\end{aligned}
$$


Taking moments from Eq. (15) (and assuming boundary terms to vanish) the following equations for their evolution can be derived:

$$
\begin{aligned}
\mathcal{P} g & =0 \\
\mathcal{P} u-\frac{1}{\mathcal{J}} \int A_{1} F \hat{\mathcal{J}} \mathrm{d} w_{1} \mathrm{~d} w_{2} & =0 \\
\mathcal{P} h-\frac{1}{\mathcal{J}} \int A_{2} F \hat{\mathcal{J}} \mathrm{d} w_{1} \mathrm{~d} w_{2} & =0 .
\end{aligned}
$$

In order to identify $u$ with $\delta f$ it is necessary to choose $A_{1}$ in such a way that Eq. (18b) can be identified with Eq. (14). This gives the condition

$$
\frac{1}{\mathcal{J}} \int A_{1} F \hat{\mathcal{J}} \mathrm{d} w_{1} \mathrm{~d} w_{2}=C\left(f_{0}, f_{0}\right)+C\left(f_{0}, \delta f\right)+\mathcal{S}-\mathcal{S}_{1} .
$$

\subsection{One-weight scheme}

Consistent with the condition given by Eq. (19) we can choose

$$
A_{1}=\frac{1}{g}\left[C\left(f_{0}, f_{0}\right)+C\left(f_{0}, \delta f\right)+\mathcal{S}-\mathcal{S}_{1}\right]
$$

leading to the weight evolution equation

$$
\dot{w}_{1, p}=\frac{1}{g}\left[C\left(f_{0}, f_{0}\right)+C\left(f_{0}, \delta f\right)+\mathcal{S}-\mathcal{S}_{1}\right] .
$$

This is the one-weight scheme proposed in Ref. [8] which suffers from the fact that the marker distribution function $g$ in general is neither known (except for some special cases) nor can be calculated with sufficient accuracy.

\subsection{Two-weight scheme}

Choosing

$$
A_{1}=\frac{w_{2}}{h}\left[C\left(f_{0}, f_{0}\right)+C\left(f_{0}, \delta f\right)+\mathcal{S}-\mathcal{S}_{1}\right]
$$

gives the weight evolution equation

$$
\dot{w}_{1, p}=\frac{w_{2, p}}{h}\left[C\left(f_{0}, f_{0}\right)+C\left(f_{0}, \delta f\right)+\mathcal{S}-\mathcal{S}_{1}\right]
$$

with the unknown quantity $h$. One can now use the freedom given by $A_{2}$ to choose

$$
A_{2}=-\frac{w_{2}}{h}\left[C\left(h, f_{0}\right)-\mathcal{S}_{1}\right]
$$

leading to the following equation for $h$ :

$$
\mathcal{P} h=\mathcal{S}_{1}-C\left(h, f_{0}\right)
$$

which is solved by $h=f_{0}$.

It is important to note that, by construction [see Eq. (17c)], the expectation 
value $(h)$ of the second weight always has to give $f_{0}$.

So, the two-weight scheme (it is identical to the one used in $[9,10]$ ) follows as

$$
\begin{aligned}
\dot{w}_{1, p} & =\frac{w_{2, p}}{f_{0}}\left[C\left(f_{0}, f_{0}\right)+C\left(f_{0}, \delta f\right)+\mathcal{S}-\mathcal{S}_{1}\right] \\
\dot{w}_{2, p} & =-\frac{w_{2, p}}{f_{0}}\left[C\left(f_{0}, f_{0}\right)-\mathcal{S}_{1}\right]
\end{aligned}
$$

together with Eqs. $(16 \mathrm{a}, 16 \mathrm{~b})$ for the trajectories of the markers. This scheme is valid for the full nonlinear equation (14) where $\dot{z}^{i}$ contains the unperturbed part $\left(\dot{z}_{0}^{i}\right)$ as well as the perturbed part $\left(\dot{z}_{1}^{i}\right)$ of the trajectories [see Eq. (2)]. One often uses linearised equations where on the left-hand side of Eq. (14), and consequently also in Eqs. $(16 \mathrm{a}, 16 \mathrm{~b})$, only the unperturbed part $\left(\mathbf{v}_{0}, \mathbf{a}_{0}\right)$ is kept, while the right-hand side contains both parts. For this case, $\mathcal{S}_{1}$ in Eq. (26b) has to be replaced by its unperturbed part, i.e. $\dot{z}_{0}^{i} \frac{\partial f_{0}}{\partial z^{i}}$.

The distribution of markers in the extended phase space can be written as a Klimontovich density

$$
F\left(\mathbf{z}, w_{1}, w_{2}, t\right)=\sum_{p} \delta\left(\mathbf{z}-\mathbf{z}_{p}\right) \delta\left(w_{1}-w_{1, p}\right) \delta\left(w_{2}-w_{2, p}\right) \hat{\mathcal{J}}^{-1}(\mathbf{z}) .
$$

Using Eq. (17) the following representations follow:

$$
\begin{aligned}
g & =\sum_{p} \delta\left(\mathbf{z}-\mathbf{z}_{p}\right) \mathcal{J}^{-1}(\mathbf{z}) \\
\delta f & =\sum_{p} w_{1, p} \delta\left(\mathbf{z}-\mathbf{z}_{p}\right) \mathcal{J}^{-1}(\mathbf{z}) \\
f_{0} & =\sum_{p} w_{2, p} \delta\left(\mathbf{z}-\mathbf{z}_{p}\right) \mathcal{J}^{-1}(\mathbf{z}) .
\end{aligned}
$$

Integrating these expressions over a small volume in phase space and eliminating it by using $g$ one finds

$$
w_{1, p}=\frac{\delta f\left(\mathbf{z}_{p}\right)}{g\left(\mathbf{z}_{p}\right)}, \quad w_{2, p}=\frac{f_{0}\left(\mathbf{z}_{p}\right)}{g\left(\mathbf{z}_{p}\right)} .
$$

These expressions can be used to define the initial conditions for Eqs. (26) at $t_{0}$. In addition, the expression to the right gives the opportunity to trace the evolution of the marker distribution $g\left(\mathbf{z}_{p}(t)\right)$ in time.

We shortly want to discuss the collisionless case, i.e. $C=0$, where it is possible to integrate Eq. (26b) yielding

$$
\frac{w_{2, p}}{f_{0}\left(\mathbf{z}_{p}\right)}=\frac{w_{2, p}\left(t_{0}\right)}{f_{0}\left(\mathbf{z}_{p}\left(t_{0}\right)\right)}=\text { const . }
$$

Substituting this into Eq. (26a) and using Eq. (29), one arrives at the following one-weight scheme:

$$
\dot{w}_{1, p}=\frac{1}{g\left(t_{0}\right)}\left[\mathcal{S}-\left(\dot{z}_{0}^{i}+\dot{z}_{1}^{i}\right) \frac{\partial f_{0}}{\partial z^{i}}\right]
$$


For $\mathcal{S}=0$ this is the standard scheme used in many applications. When the system is initially in an equilibrium state $\dot{z}_{0}^{i} \frac{\partial f_{0}}{\partial z^{i}}$ vanishes naturally. If this is not the case this term is, nevertheless, often neglected (see e.g. [19]), what can now be seen to be equivalent to introducing an artificial source given by $\mathcal{S}=\dot{z}_{0}^{i} \frac{\partial f_{0}}{\partial z^{i}}$ in Eq. (1).

\section{Properties of the two-weight scheme}

To understand the properties of the two-weight scheme we use a one-dimensional test problem: Ornstein-Uhlenbeck diffusion [20]

$$
\frac{\partial f}{\partial t}=\mu \frac{\partial}{\partial v}(v f)+\frac{1}{2} D^{2} \frac{\partial^{2}}{\partial v^{2}} f
$$

Although this process is relatively simple, it shares important features with more complicated physical applications. For the initial condition $f(v, t=0)=G(a)$ with $G(a)=\frac{a}{\sqrt{\pi}} e^{-a^{2} v^{2}}$ it has the solution

$$
f_{\mathrm{OU}}(v)=G\left(\frac{1}{h}\right), h^{2}(t)=\frac{D^{2}}{\mu}\left[1-e^{-2 \mu t}\left(1-\frac{\mu}{a^{2} D^{2}}\right)\right]
$$

which for large times approaches the stationary solution $f_{\mathrm{S}} \stackrel{\text { def }}{=} G(\sqrt{\mu} / D)$. For $\mu=0$ the system has no drift and only diffuses, so the stationary state is $f_{\mathrm{OU}}=0$. For $\mu=4$, the other limiting case, the system does not evolve at all since the stationary state coincides with the initial condition. So, increasing $\mu \in[0,4]$ leads to less evolution away from the initial conditions.

The function $f_{0}$ in the $\delta f$ ansatz and the initial marker distribution function are chosen as $f_{0}=G\left(b_{0}\right)$ and $g=G\left(b_{\mathrm{g}}\right)$ with free constants $b_{0}, b_{\mathrm{g}}$. The scheme follows from Eqs. (26) and Eq. (16b) with $C\left(f_{0}, \delta f\right)=0, \mathcal{S}=0, \mathcal{S}_{1}=0$ and $\mathbf{S}=-\mu v, \mathbf{D}=D$. The integration of the stochastic differential equation for the trajectories is done using an Euler-Maruyama scheme [21]. The basic parameters for the simulations are $N=4 \cdot 10^{4}, D=1, a=b_{0}=b_{\mathrm{g}}=2$ and a time-step of $\Delta t=5 \cdot 10^{-3}$ (the value of $\mu$ will be given later). For comparison, we also perform full- $f$ simulations for the problem by the standard procedure, i.e. applying the basic theorem directly to Eq. (32). Then, $f$ is directly represented by the distribution function of the markers (importance sampling) that are advanced using Eq. (16b) (note, that for a full- $f$ scheme the weights are constant in time). The initial conditions are directly represented by the distribution of the markers at $t=0$ which must be equal to $f(v, 0)$.

In the simulations, the numerical distribution function $\hat{f}_{i}$ is obtained by binning the markers into $N_{\mathrm{b}}$ bins of width $\Delta v$ centered at $v_{i}$. For doing this, it is convenient to define a binning function $\Lambda_{i}(v)=\frac{1}{\Delta v}\left[H\left(v-v_{i}+\frac{\Delta v}{2}\right)-H\left(v-v_{i}-\frac{\Delta v}{2}\right)\right]$. While for full- $f$ simulations only the markers have to be binned, the weights $w_{1}$ have to be taken into account in the $\delta f$ simulations giving $\widehat{\delta f}_{i}=\int \Lambda_{i} \delta f \mathrm{~d} v=$ $E\left(\Lambda_{i} \frac{\delta f}{g}\right) \approx \frac{1}{N} \sum_{p=1}^{N} w_{1, p} \Lambda_{i}\left(v_{p}\right)$ and finally $\hat{f}_{i}=f_{0}\left(v_{i}\right)+\widehat{\delta f}_{i}$. The error $\epsilon$ of the 
result is then defined by

$$
\epsilon^{2}=\frac{1}{N_{\mathrm{b}}} \sum_{i=1}^{N_{\mathrm{b}}}\left[f_{\mathrm{OU}}\left(v_{i}\right)-\hat{f}_{i}\right]^{2} .
$$

The error as a function of time for the full- $f$ and $\delta f$ scheme is shown in Figure 1 where we have chosen $\mu=0$ and $\mu=3$, respectively. As a time reference, we give the value $t_{1 / 2}$, i.e. the time at which the solution at $v=0$ has decayed to half-way between the initial and the final state. One sees that, as expected, the $\delta f$ scheme starts with a vanishing error at $t=0$ but the error grows with time and eventually exceeds the time-independent error of the full- $f$ scheme. In the case $\mu=0, f$ evolves constantly away from $f_{0}$ and one may argue that this could be the reason for the error to increase with time. However, this is not the reason, as it can be seen for the case $\mu=3$. Here, the final state is essentially reached at $t \approx 0.5$, i.e. the difference $f-f_{0}$ stays nearly constant for later times. Nevertheless, the error is still increasing, but at a slower rate than for $\mu=0$. This is a consequence of the fact that the $\delta f$ scheme should become better if the difference between $f_{0}$ and the final state becomes smaller (since then $\delta f \ll f_{0}$ is better fulfilled). Choosing $\mu=4$ gives no evolution of the system at all, since the initial state is equal to the final state (the error of the $\delta f$ scheme then is exactly zero in contrast to the full- $f$ scheme, which still has a finite error). Consequently for $\mu \approx 4$ the growth of the error is very small and the $\delta f$ scheme is better than the full- $f$ scheme for a reasonably long time (e.g. for $\mu=3.9$ the errors of both schemes become similar at $t \approx 20$ ).

In order to get more insight into the reason for this behaviour (also called weight spreading [9]) it is sufficient to use Eqs. (16b, 26b) which, for the present model problem, can be written as

$$
\dot{w}_{2, p}=G\left(v_{p}\right) w_{2, p}, \quad \dot{v}_{p}=-\mu v_{p}+D \frac{\mathrm{d} W}{\mathrm{~d} t}
$$

with $G(v)=\left(\mu-b_{0}^{2} D^{2}\right)\left(2 b_{0}^{2} v^{2}-1\right)$. The solution for $v$ can be expressed by Itoh integrals and the expectation value of $G$ can then be calculated, giving $E(G)=$ $-\frac{1}{\mu}\left(\mu-b_{0}^{2} D^{2}\right)^{2}$ for large times. Since $E(G)<0$, it follows that all the $w_{2, p}$ will eventually evolve towards zero, the fixed point of the first equation in Eqs. (35). Even if $f$ does no longer change because it has reached a stationary state, the weights $w_{2, p}$ are still evolving, but with growing time more and more of them will no longer contribute since their value is approaching zero. Nevertheless, the expectation value of $w_{2}$ in a velocity bin always has to give $f_{0}$, which is only possible if the values of the remaining $w_{2, p}$ grow with time. So less and less $w_{2, p}$ with constantly increasing value are available and, consequently, the noise in the expectation value gets larger. This process for the second weight $w_{2}$ is then transferred by Eq. (26a) to the first weight $w_{1}$ and to $\delta f$. This behaviour of the $w_{2}$ is illustrated in Figure 2 where the probability distribution function for the $w_{2}$ is shown at different times ( $\mu=3$ has been used). Since $g=f_{0}$ has been chosen at $t=0$, all the weights initially have the same value. The distribution 
then rapidly spreads with time and develops a pronounced maximum near the fixed point $w_{2}=0$.

\section{Improving the two-weight scheme}

An error increasing with time is undesirable for a numerical scheme. Instead one would prefer a scheme which behaves as a $\delta f$ scheme for early times and for later times has an error bounded by that of the full- $f$ scheme.

We now define the following quantity $c_{p}(t)=w_{1, p}(t)+w_{2, p}(t)$. The first weight can then be expressed as

$$
w_{1, p}(t)=c_{p}(t)-w_{2, p}(t)
$$

having the form of a control variate formulation but now for the collisional case. If $C\left(f_{0}, \delta f\right)$ is neglected and $\mathcal{S}$ is assumed to be zero, then Eqs. (26) can be added to give a constant of motion: $c_{p}(t)=c_{p}\left(t_{0}\right)$. As a consequence, Eq. (36) has the same form as Eq. (11). It follows, that the equation for $w_{2, p}(t)$ just describes one of the possible stochastic processes with an expectation value $f_{0}$ which is then used as a CV. (One may speculate that an improved scheme could be constructed by using a better suited stochastic process as a CV. This process also has to give $f_{0}$ but should not have a fixed point at $w_{2}=0$.). This kind of $\mathrm{CV}$, as it follows from the $\delta f$ formulation, we call 'ordinary control variate', since it does not make use of the full possibilities provided by the control variate method.

We recall the general definition of a control variate (see e.g. [22, 23]): Assume that the expectation value $\mathrm{E}(X)$ of a stochastic process $X$ is sought after, where the variance $\mathrm{V}(X)$ is a measure of its error. Introduce a second process $Y$, called control variate, with known expectation value $\mathrm{E}(Y)=y$ and define a new process by

$$
\tilde{X}=X-\alpha(Y-y),
$$

with $\alpha$ a parameter yet to be determined. Evidently, for this process $\mathrm{E}(\tilde{X})=$ $\mathrm{E}(X)$. Its variance $\mathrm{V}(\tilde{X})$ as a function of $\alpha$ is minimised by choosing

$$
\alpha=\frac{\operatorname{Cov}(X, Y)}{\mathrm{V}(Y)}
$$

(with $\operatorname{Cov}(X, Y)$ the covariance between $X$ and $Y$ ). Therefore, by using a CV, one can construct a new process with smaller variance, where $\alpha$ can be calculated by the usual estimators for the covariance and variance.

This scheme includes the ordinary $\mathrm{CV}$ as a special case. To see this, one has to use the fact that the $\delta f$ approach only improves the statistics under the assumption that $\delta f \ll f_{0}$. In the above formulation, this means that $X \approx Y$ corresponding to $\alpha \approx 1$. This shows that the $\delta f$ approach viewed as a CV is not optimal.

We now use the idea of the general CV to construct an improved scheme for the simulation of collisions. The quantity of interest is the binned distribution 
function $\hat{f}_{i}=E\left(\Lambda_{i} \frac{f}{g}\right)$ obtained from the process $X=\Lambda_{i} \frac{f}{g}$. In order to improve the estimator we construct the following process (setting $Y=\Lambda_{i} \frac{f_{0}}{g}$ ):

$$
\tilde{X}=\Lambda_{i} \frac{f}{g}-\alpha_{i}\left(\Lambda_{i} \frac{f_{0}}{g}-F_{0, i}\right) \text {, }
$$

with $F_{0, i}=\int \Lambda_{i} f_{0} \mathrm{~d} v \approx f_{0}\left(v_{i}\right)$ the known expectation value. Using $c_{p}$ and $w_{2, p}$ it follows that

$$
\hat{f}_{i}=E(\tilde{X}) \approx \frac{1}{N} \sum_{p=1}^{N} \Lambda_{i}\left(c_{p}-\alpha_{i} w_{2, p}\right)+\alpha_{i} F_{0, i}
$$

This motivates the introduction of a new weight $\tilde{w}_{1, p}$ [compare with Eq. (36)] defined by

$$
\tilde{w}_{1, p}(t)=c_{p}(t)-\alpha_{i} w_{2, p}(t)
$$

Here $\alpha_{i}$ in each bin is given by

$$
\alpha_{i}=\frac{\operatorname{Cov}\left(c, w_{2}\right)}{\mathrm{V}\left(w_{2}\right)},
$$

where the usual estimators are used for the covariance and the variance.

For the example used here, $c_{p}$ is a constant of motion and thus the introduction of $\alpha_{i}$ can be interpreted as a way to smoothly interpolate between the two limiting cases $\alpha_{i}=0$ and $\alpha_{i}=1$, corresponding to the full- $f$ and the $\delta f$ scheme, respectively. Also $\alpha_{i}$ is a measure for the quality of the CV: If $\alpha_{i} \approx 1$ the solution is very well approximated by the CV but if $\alpha_{i} \approx 0$ a CV does not lead to any improvement.

If there are not sufficient markers in a bin it is difficult to estimate the covariance or the variance accurately. This leads to unreasonable values for $\alpha_{i}$. We have found that in these cases, it is necessary to introduce a cutoff function for $\alpha_{i}$, which guarantees $\alpha_{i} \in[0,1]$ : For example, if there are no markers in a bin, $\widehat{\delta f}_{i}$ cannot be estimated. Then, the best one can do is to use $f_{0}$ for approximating $\hat{f}_{i}$ and consequently $\alpha_{i}=1$ is chosen. Also, if $\alpha_{i}>1$, what may happen if e.g. the variance is very small, it suffices to artificially set it to one.

Figure 3 shows two runs for the same parameters used for Fig. 1 but now employing the new scheme. It can be seen that the error of the new scheme initially behaves as for the $\delta f$ scheme, i.e. the error increases with time, but after some time it stops growing and eventually follows the level set by the full$f$ scheme. Therefore, the error of the new scheme is always lower than the error of the $\delta f$ and the full- $f$ scheme, respectively. Note that in this test problem, the underlying dynamics of the system is not affected. The CV only serves to improve the estimation of the expectation values calculated to approximate the solution. Nevertheless, in a more realistic application (e.g. in plasma physics), the solution couples back to the system through the field equations and so the accuracy of the moments is essential. 


\section{Application to nonlinear collisionless simulations}

In this section we illustrate the application of an enhanced CV for a more elaborate problem: The simulation of collisionless turbulence driven by the iontemperature-gradient instability (ITG) using the TORB code. This code solves the nonlinear electrostatic collisionless gyrokinetic equation for ions (assuming adiabatic electrons) globally in a cylindrical configuration. The classical PIC approach (one-weight scheme) is combined with a B-spline discretisation of the field equation. A detailed description of the code and the equations solved can be found in [15]. The numerical parameters from this reference (with the exception of $\kappa_{\mathrm{T}_{\mathrm{i}}}=3.5$ ) are also used in the following simulation which in addition includes the correct treatment of the zonal flow (see e.g. [24]).

Starting from Eq. (39), where now $\Lambda_{i}$ denotes a B-spline function, and using Eqs. $(37,38)$ one arrives at

$$
\alpha_{i}=\frac{\operatorname{Cov}\left(\Lambda_{i} \frac{f}{g}, \Lambda_{i} \frac{f_{0}}{g}\right)}{\mathrm{V}\left(\Lambda_{i} \frac{f_{0}}{g}\right)} .
$$

The implementation into the TORB code is straightforward and can be done with little overhead in computational time since the calculation of the necessary estimators can be combined with the calculation of the density in the general charge assignment procedure. In a subsequent step the density is corrected correspondingly by using $\alpha_{i}$ before the field equation is solved.

In such a case, $\alpha_{i}$ is a function of the three space coordinates. Its maximum and minimum values as a function of time are shown in Figure 4 . In the linear phase $(t<0.1 \mathrm{~ms})$ its value is, as expected, very close to one since the classical $\mathrm{CV}$ is expected to work very well. Only in the nonlinear phase, a significant deviation from one is to be expected. This is indeed the case but the variation in $\alpha_{i}$ is still relatively small (a few percent). This means that also in the nonlinear phase the classical CV is still a very good choice and consequently the inclusion of the $\alpha_{i}$-feedback does not lead to a significant improvement of the simulation results. Nevertheless, $\alpha_{i}$ is still a useful diagnostic quantity allowing to quantify how good the CV of the classical $\delta f$ method actually is (the small deviation of $\alpha_{i}$ from one seems to be specific for this example and can be probably quite different in other cases).

A second example for the application of an enhanced $\mathrm{CV}$ is the implementation of particle number conservation in a $\delta f$ scheme. The total (physical) particle number $N_{\mathrm{ph}}=\int f \mathrm{~d} \mathbf{z}$ in the volume should be conserved what is equivalent to $\delta N_{\mathrm{ph}}=\int \delta f \mathrm{~d} \mathbf{z}=0$ for all times. Using the usual estimator, this can be written as $\delta N_{\mathrm{ph}}=E(w)=\frac{1}{N} \sum_{p=1}^{N} w_{p}$ (where again a weight $w=\frac{\delta f}{g}$ has been introduced). It is a general problem for classical $\delta f$ simulations that $\delta N_{\mathrm{ph}}$ starts deviating from zero with time (although this deviation gets smaller with increasing particle number it is an undesirable behaviour which can cause problems) and consequently the system gains or looses charge. Figure 5 (upper part, dashed curve), where the time development of $\delta N_{\mathrm{ph}}$ is depicted, shows 
that when the system enters the nonlinear phase the total particle number decreases. Parallel to this the field energy shows a growth after $t \approx 0.25 \mathrm{~ms}$ seen in Figure 5 (lower part, dashed curve). Since this growth is not compensated by a decrease in kinetic energy it leads to a violation of the total energy of the system.

We now construct an enhanced $\mathrm{CV}$ which ensures a constant particle number: The fact that $\delta N_{\mathrm{ph}}=E(w)$ must be zero can now be used to construct a $\mathrm{CV}$, i.e. one gets a new process by setting $X=\Lambda_{i} w, Y=w, y=0$ in Eq. (37). Using Eq. (38) the result is

$$
\tilde{X}=\Lambda_{i} w-\frac{E\left(\Lambda_{i} w^{2}\right)-E\left(\Lambda_{i} w\right) E(w)}{E\left(w^{2}\right)-E(w)^{2}} w
$$

giving

$$
E(\tilde{X})=E\left(\Lambda_{i} w\left[1-\frac{w-E(w)}{E\left(w^{2}\right)-E(w)^{2}} E(w)\right]\right) .
$$

This motivates the definition of an effective weight, which can be implemented very efficiently,

$$
\tilde{w}=w \frac{E\left(w^{2}\right)-w E(w)}{E\left(w^{2}\right)-E(w)^{2}}
$$

used for calculating the expectation values. By construction $\delta N_{\mathrm{ph}}=E(\tilde{w})=0$ is now guaranteed. A significant improvement is seen in the electrostatic field energy (Figure 5, lower part, solid curve): Its rapid growth for later times has disappeared and it now stays nearly constant leading to a much better energy conservation.

\section{Conclusions}

The $\delta f$ approach helps to decrease the statistical noise by several orders of magnitude in PIC simulations of collisionless plasmas. Including collisional effects into this approach is possible by working in an extended phase space and then deriving equations for the time evolution of the moments. While the two-weight scheme initially shows the expected behaviour, i.e. having a much smaller error than a full- $f$ scheme, it has the drawback that the statistical error increases indefinitely with time. The behaviour of the numerical scheme can be investigated for a simple model that is not obfuscated by too many physical details. For this reason, we have chosen the Ornstein-Uhlenbeck diffusion as a basic model problem. It is simpler than more realistic problems but, still, shares the property of relaxing towards a stationary state. For this model, the behaviour of the usual two-weight scheme could be demonstrated and clarified. We have shown that under some assumptions the scheme can be regarded as an 'ordinary control variate' scheme, which is also the basis of the collisionless $\delta f$ approach. It is thus possible to improve the scheme by using a more general control variate, where a free parameter is used to automatically control how much 
of the control variate is used in the calculation. This approach makes it possible to construct an improved scheme that smoothly switches from a $\delta f$ to a full- $f$ type behaviour, with the consequence that the advantages of both approaches with respect to the statistical error could be combined: Initially, the error is small since the solution is well approximated by the control variate while, for later times, a full- $f$ approach gives a comparatively smaller error than the $\delta f$ scheme. The presented scheme can be applied to more realistic situations where the term $C\left(f_{0}, \delta f\right)$ is retained. This issue and applications to more complicated cases is currently under study.

Using a general control variate is also useful for collisionless simulations either as a diagnostic tool to trace the quality of the initially chosen $\mathrm{CV}$ over the lifetime of the simulation or to be used in a feedback procedure to automatically adapt the control variate to the evolving solution. Therefore, the presented scheme is a step towards an adaptive control variate scheme. Also other known (global) constraints on the system, as e.g. the particle number conservation, can be used as a CV thus forcing the simulation to adhere to it. In case of the implementation of the particle number conservation the $n=0, m=0$ mode and thus the zonal flow of the nonlinear system acts much more realistic which leads to a much better quality of the field energy and consequently to much better energy conservation. In addition, it may be possible to further adapt the CV to also include energy conservation.

Since an enhanced CV can be implemented with a small overhead in computational time it becomes a valuable technique in the toolbox of Monte Carlo methods and can be useful for a variety of problems.

\section{Acknowledgments}

We would like to thank S. Brunner and A. Bottino for helpful discussions.

\section{Appendix A. Global variance minimization with multiple control vari- ates}

Following Lavenberg and Welch [22] one can introduce $N_{\mathrm{b}}$ control variates. Thus one starts from a generalisation of Equation (40):

$$
E\left(\Lambda_{i} \frac{f}{g}\right)=E\left(\Lambda_{i} \frac{f}{g}\right)-\boldsymbol{\alpha}\left[E\left(\Lambda \frac{f_{0}}{g}\right)-\mathbf{F}\right]=E\left(\Lambda_{i} \frac{f}{g}\right)-\boldsymbol{\alpha} E(\mathbf{K}) .
$$

The vector components are the individual binning functions $\Lambda_{i}$ and $F_{0, i}$ (also the vector $\mathbf{K}=E\left(\boldsymbol{\Lambda} f_{0} / g\right)-\mathbf{F}$ has been introduced). The vector $\boldsymbol{\alpha}$ can be obtained by minimisation of the variance of $\Lambda_{i} \frac{f}{g}$. The solution is

$$
\boldsymbol{\alpha}=\left[E\left(\mathbf{K K}^{\mathrm{T}}\right)-E(\mathbf{K}) E\left(\mathbf{K}^{\mathrm{T}}\right)\right]^{-1}\left[E\left(\Lambda_{i} \frac{f}{g} \mathbf{K}\right)-E\left(\Lambda_{i} c\right) E(\mathbf{K})\right]
$$

where the first quantity in square brackets, the covariance matrix, will be denoted by $\mathbf{M}_{\mathrm{c}}$. Note, that the minimisation of the variance has been performed 
over all the bins, not as before only separately for each bin. This formula hides considerable complexity as it connects each bin with all others. The matrix elements of $\mathbf{M}_{\mathrm{c}}$ are subject to noise because they will be computed from a stochastic process. By error propagation, noise from some 'contaminated' bins may invalidate the calculation. Thus the calculation of $\mathbf{M}_{\mathrm{c}}^{-1}$ is the most critical point of the method.

In the following, we show that $\boldsymbol{M}_{\mathrm{c}}^{-1}$ can be written in a way that avoids a numerically expensive matrix inversion. After some algebra (and replacing the expectation value by the usual estimator) one finds

$$
\left(\mathbf{M}_{\mathrm{c}}\right)_{i j} \approx \frac{1}{N} \sum_{p=1}^{N} \Lambda_{i} \delta_{i j} w_{2, p}^{2}-F_{0, i} F_{0, j}
$$

what can be written as $\mathbf{M}_{\mathrm{c}}=\mathbf{D}-\mathbf{F} \mathbf{F}^{\mathrm{T}}$ with $D_{i j}=d_{i} \delta_{i j}\left(d_{i}>0\right)$. The inverse can be obtained from the Sherman-Morrison formula

$$
\mathbf{M}_{\mathrm{c}}=\mathbf{D}+\frac{\mathbf{D}^{-1} \mathbf{F} \mathbf{F}^{\mathrm{T}} \mathbf{D}^{-1}}{1-\mathbf{F}^{\mathrm{T}} \mathbf{D}^{-1} \mathbf{F}}
$$

leading to

$$
\left(\mathbf{M}_{\mathrm{c}}^{-1}\right)_{i j}=d_{i}^{-1} \delta_{i j}+\frac{d_{i}^{-1} F_{i} F_{j} d_{j}^{-1}}{1-\sum_{k=1}^{N_{\mathrm{b}}} F_{k} d_{k}^{-1} F_{k}} .
$$

Obviously, this formula only holds if $1-\sum_{k} F_{k} d_{k}^{-1} F_{k} \neq 0$. Therefore, the control variates have to be carefully chosen so that the latter condition is fulfilled. Actually, the matrix $\mathbf{M}_{\mathrm{c}}$, being a covariance matrix, is positive semidefinite. However, as the variance is calculated using an estimator for a particular realization of a stochastic process, the definiteness of the matrix is not longer guaranteed. Therefore, it is a difficult task to perform a global optimisation. The control variates have to be properly chosen and the quality of the variance calculation has to be controlled during the simulation. In the local case, this fact reflects itself in the cut-off procedure for the $\alpha_{i}$. Further research is needed to determine how a global variance minimisation algorithm can be effectively implemented.

Note, that for the case considered here, it is straightforward to show that if $d_{i} \gg F_{i}^{2}$ holds for all $i$, global and local results agree.

\section{References}

[1] C. K. Birdsall, A. B. Langdon, Plasma Physics via Computer Simulation, McGraw-Hill, 2004.

[2] R. W. Hockney, J. W. Eastwood, Computer Simulation using Particles, Institute of Physics, 1988.

[3] T. S. Hahm, Nonlinear gyrokinetic equations for tokamak microturbulence, Phys. Fluids 31 (1988) 2670. 
[4] M. Kotschenreuther, Numerical simulation, Bull. Am. Phys. Soc. 33 (1988) 2107.

[5] A. Y. Aydemir, A unified monte carlo interpretation of particle simulations and applications to non-neutral plasmas, Phys. Plasmas 1 (1994) 822.

[6] S. J. Allfrey, R. Hatzky, A revised $\delta f$ algorithm for nonlinear PIC simulation, Comp. Phys. Commun. 154 (2003) 98.

[7] R. Hatzky, A. Könies, A. Mishchenko, Electromagnetic gyrokinetic PIC simulation with an adjustable control variates method, J. Comp. Phys. 225 (2007) 568.

[8] Y. Chen, R. B. White, Collisional $\delta f$ method, Phys. Plasmas 4 (1997) 3591.

[9] S. Brunner, E. Valeo, J. A. Krommes, Linear delta-f simulations of nonlocal electron heat transport, Phys. Plasmas 6 (1999) 4504.

[10] W. X. Wang, N. Nakajima, M. Okamoto, S. Murakami, Global $\delta f$ particle simulation of neoclassical transport and ambipolar electric field in general geometry, Plasma Phys. Controlled Fusion 41 (1999) 1091.

[11] P. Helander, D. J. Sigmar, Collisional Transport in Magnetized Plasmas, Cambridge University Press, 2002.

[12] M. N. Rosenbluth, W. M. MacDonald, D. L. Judd, Fokker-planck equation for an inverse-square force, Phys. Rev. 107 (1957) 1.

[13] X. Q. Xu, M. N. Rosenbluth, Numerical simulation of ion-temperaturegradient-driven modes, Phys. Fluids B 3 (1991) 627.

[14] G. Hu, J. A. Krommes, Generalized weighting scheme for $\delta f$ particlesimulation method, Phys. Plasmas 1 (1994) 863.

[15] R. Hatzky, T. M. Tran, A. Könies, R. Kleiber, S. J. Allfrey, Energy conservation in a nonlinear gyrokinetic particle-in-cell code for ion-temperaturegradient-driven modes in $\theta$-pinch geometry, Phys. Plasmas 9 (2002) 898.

[16] R. Graham, Covariant formulation of non-equilibrium statistical thermodynamics, Z. Physik B 26 (1977) 397.

[17] H. Risken, The Fokker-Planck Equation, Springer, 1996.

[18] Z. Lin, W. M. Tang, W. W. Lee, Gyrokinetic particle simulation of neoclassical transport, Phys. Plasmas 2 (1995) 2975.

[19] P. Angelino, A. Bottino, R. Hatzky, S. Jolliet, O. Sauter, T. M. Tran, L. Villard, On the definition of a kinetic equilibrium in global gyrokinetic simulations, Phys. Plasmas 13 (2006) 052304.

[20] G. E. Uhlenbeck, L. S. Ornstein, On the theory of the brownian motion, Physical Review 36 (1930) 823. 
[21] P. E. Kloeden, E. Platen, Numerical Solution of Stochastic Differential Equations, Springer, 1999.

[22] S. S. Lavenberg, P. D. Welch, A perspective on the use of control variables to increase the efficiency of monte carlo simulations, Management Science 27 (1981) 322.

[23] P. Glasserman, Monte Carlo Methods in Financial Engineering, Springer, 2003.

[24] S. Jolliet, B. F. McMillan, T. Vernay, L. Villard, R. Hatzky, A. Bottino, P. Angelino, Influence of the parallel nonlinearity on zonal flows and heat transport in global gyrokinetic particle-in-cell simulations, Phys. Plasmas 16 (2009) 072309. 


\section{Captions}

Figure 1: Time development of the error for $\mu=0$ (upper) and $\mu=3$ (lower) for the full- $f$ scheme (dotted) and the two-weight scheme (solid). The reference times are $t_{1 / 2}=0.375$ and $t_{1 / 2}=0.1$, respectively.

Figure 2: Distribution function of the weight $w_{2}$ for different times $(\mu=3)$.

Figure 3: Solid: Time development of the error for $\mu=0$ (upper) and $\mu=3$ (lower) for the improved scheme. For reference the same data as in Figure 1 are also shown (dashed, dotted).

Figure 4: Spatial maximum (solid) and minimum (dashed) value of $\alpha$ over time.

Figure 5: Particle number variation over time (upper) and electrostatic field energy (lower) for a run without (dashed) and with correction (solid). 


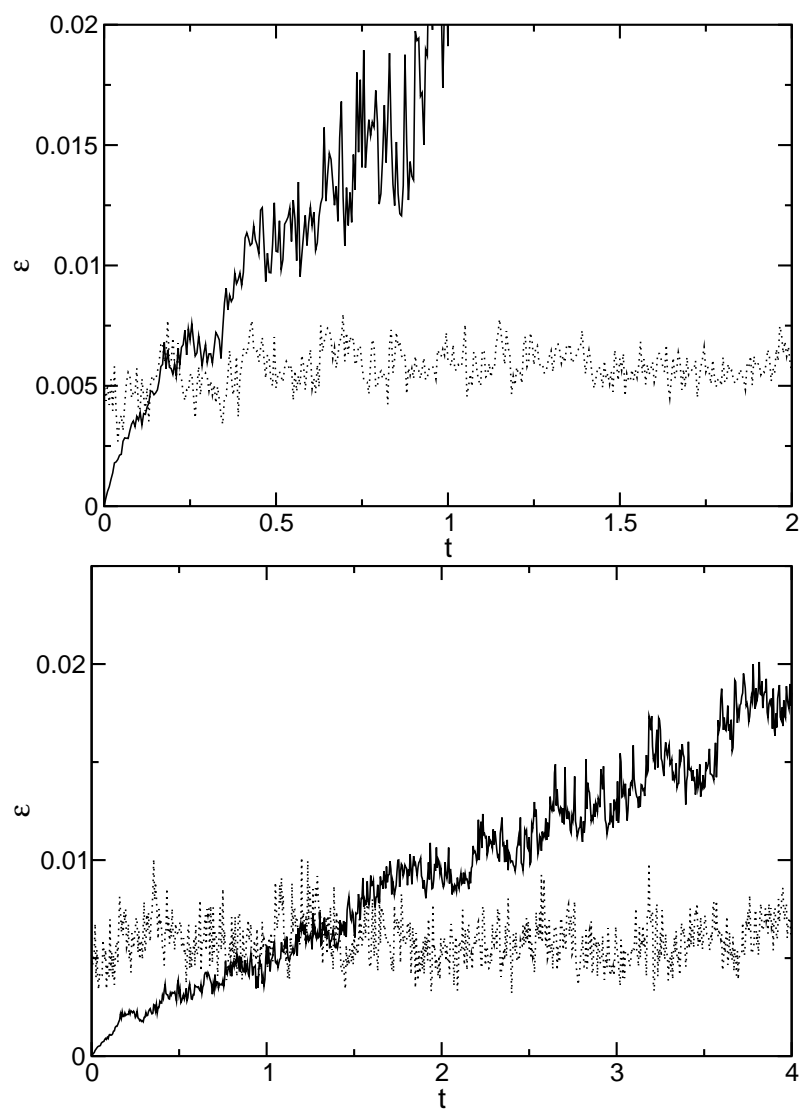

Figure 1 


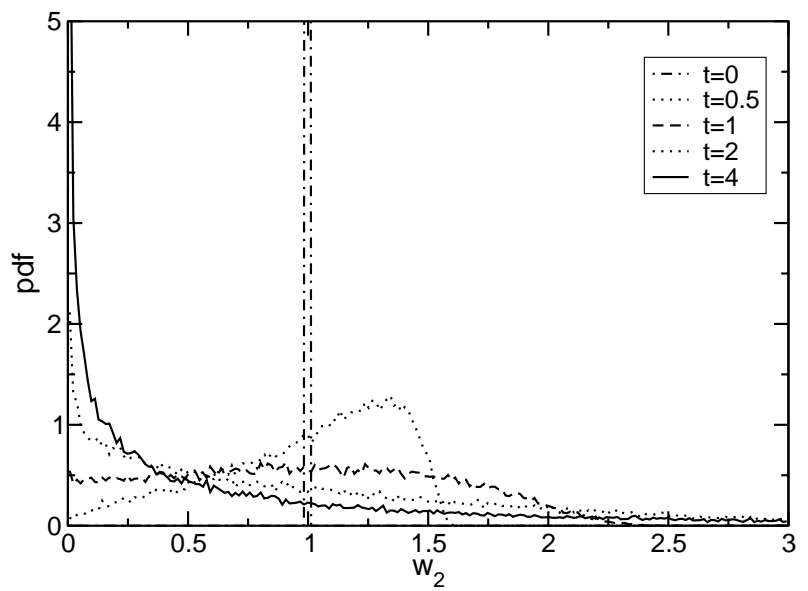

Figure 2 


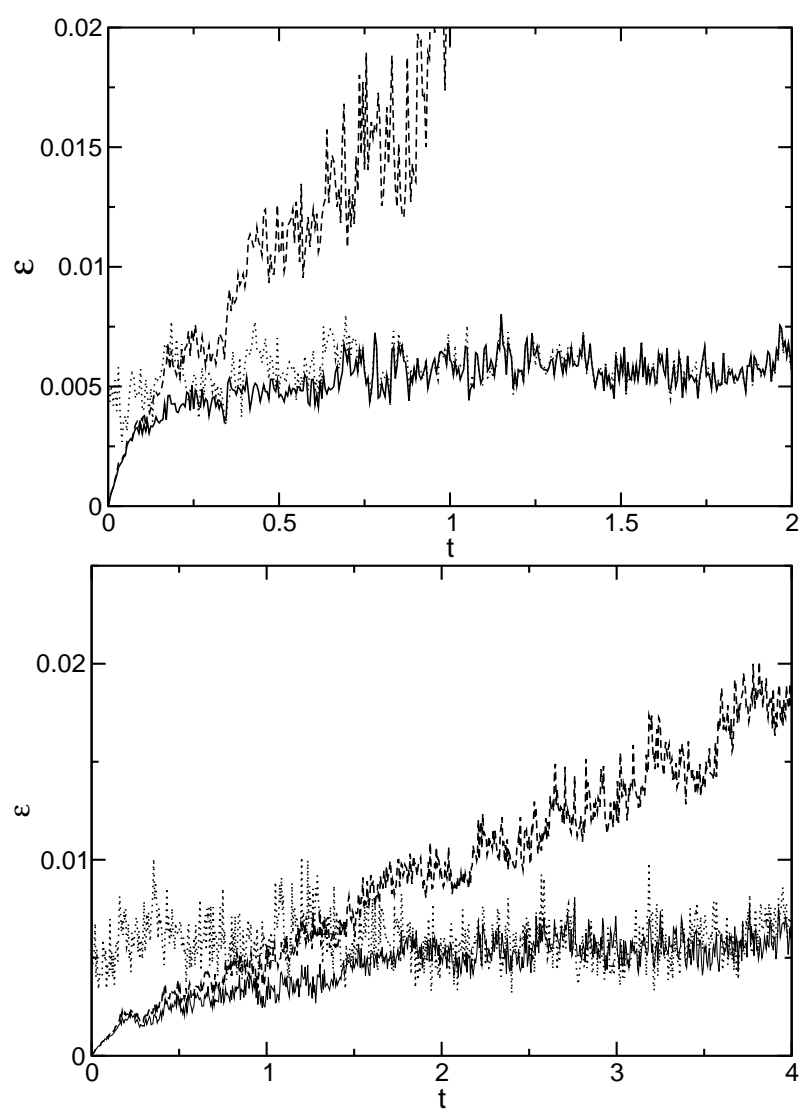

Figure 3 


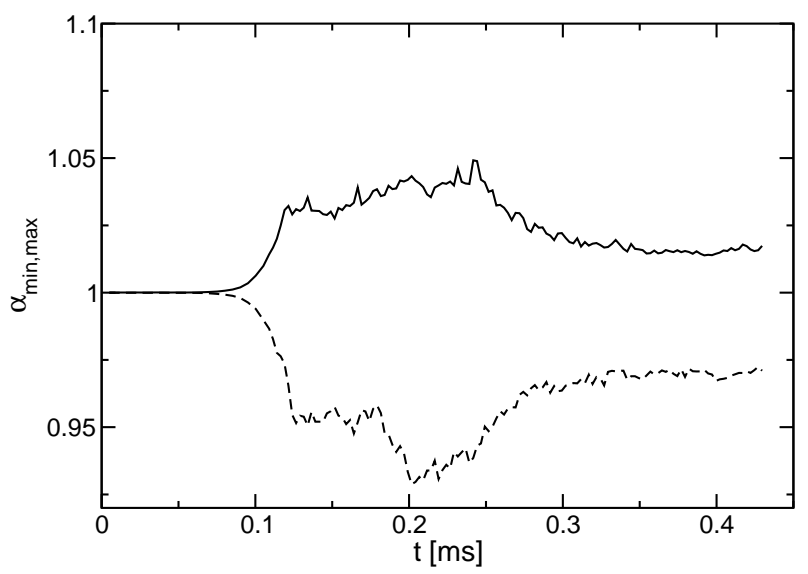

Figure 4 

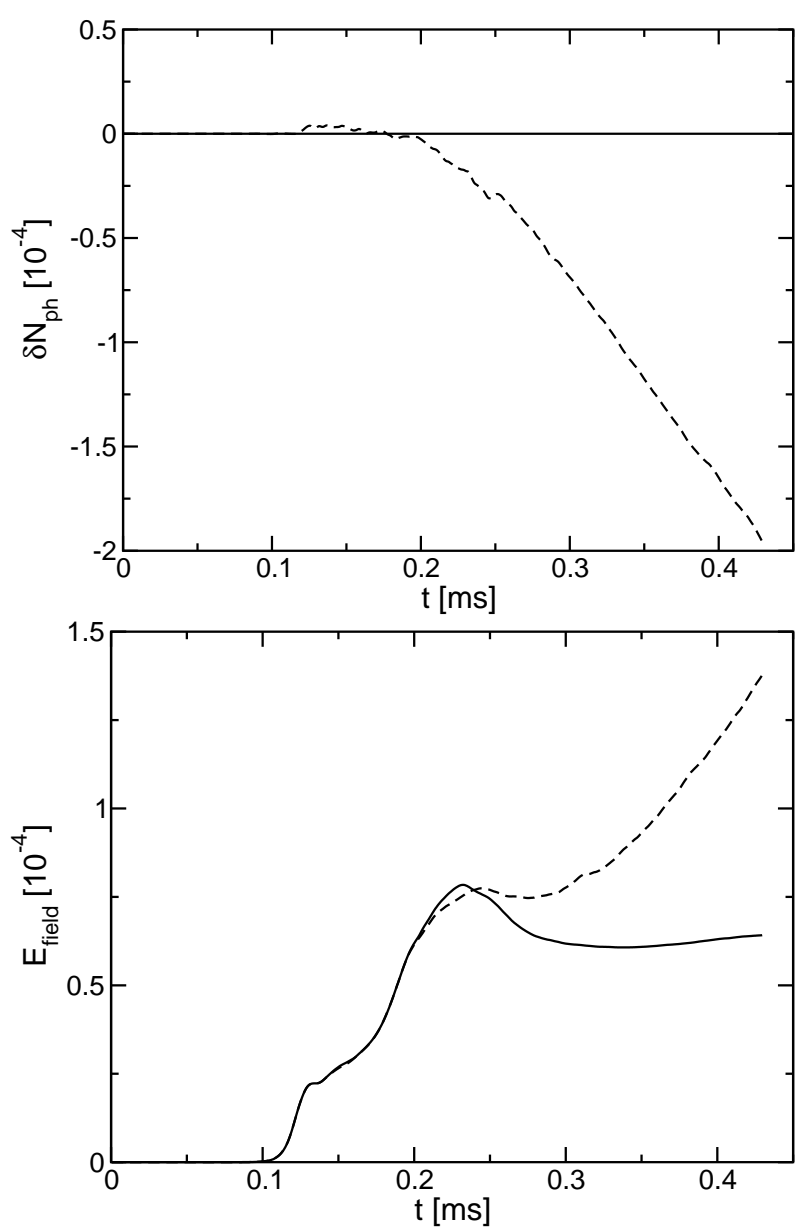

Figure 5 